\title{
Universally conserved and yeast-specific U1 snRNA sequences are important but not essential for U1 snRNP function
}

\author{
Xiaoling Liao, Leo Kretzner, ${ }^{1}$ Bertrand Seraphin, ${ }^{2}$ and Michael Rosbash \\ Howard Hughes Medical Institute, Department of Biology, Brandeis University, Waltham, Massachusetts 02254 USA
}

\begin{abstract}
To study the contribution of the large, 568-nucleotide yeast (Saccharomyces cerevisiae) U1 snRNA to premRNA splicing, we generated mutations in two regions of the molecule and introduced each mutant gene back into yeast as the sole copy of the U1 snRNA gene. We mutagenized the "A loop," a subregion highly conserved in primary sequence in all U1 snRNA molecules analyzed to date. We also mutagenized a portion of the yeast core subdomain, a region conserved in primary and secondary structure among several yeast species but absent from the much smaller metazoan U1 molecule. Surprisingly, mutations in these two regions had little or no effect on growth rate, yet several of them affected an inefficiently spliced reporter gene construct. In addition, combinations of mutants in both regions gave rise to reduced growth rates. Using the latter assay, we confirmed some of the proposed secondary structure of the yeast core domain. The experiments indicate that both regions contribute to U1 snRNP activity but that mutations in a single region do not have a substantial effect on growth rate because $\mathrm{U} 1 \mathrm{snRNP}$ activity is not rate-limiting for growth.
\end{abstract}

[Key Words: U1 snRNA; pre-mRNA splicing; Saccharomyces cerevisiae]

Received June 22, 1990; revised version accepted August 9, 1990.

Pre-mRNA splicing takes place in a large multisubunit complex termed the spliceosome (Brody and Abelson 1985; Frendewey and Keller 1985; Grabowski et al. 1985). A variety of protein factors and four small nuclear ribonucleoprotein particles (snRNPs) are present in spliceosomes and/or participate in pre-mRNA splicing. Each of these snRNPs, U1, U2, U5, and U4/U6, contains a single small nuclear RNA (snRNA) molecule (two snRNAs in the case of U4/U6) and 7-10 proteins (for reviews, see Maniatis and Reed 1987; Guthrie and Patterson 1988; Steitz et al. 1988). The analysis of in vitro spliceosome formation by native gel electrophoresis, as well as a variety of lines of evidence, indicates a stepwise assembly process that is very similar in extracts derived from yeast (Saccharomyces cerevisiae) and from mammalian cells (Konarska and Sharp 1986, 1987; Pikielny et al. 1986; Cheng and Abelson 1987; Lamond et al. 1988).

In the yeast in vitro system, the interaction of U1 snRNP with the pre-mRNA precedes, and is necessary for, the subsequent association of the other three snRNPs (Ruby and Abelson 1988; Seraphin et al. 1988; Seraphin and Rosbash 1989a). As in mammalian cells (Zhuang and Weiner 1986), this interaction is dependent on base-pairing between the $5^{\prime}$ end of Ul snRNA and the 5' splice site (Seraphin et al. 1988; Siliciano and Guthrie 1988). This pre-mRNA-Ul snRNP complex has been called a commitment complex, because as the earliest

Current addresses: ${ }^{1}$ Fred Hutchinson Cancer Research Center, Seattle, Washington 98104 USA; ${ }^{2}$ Institut Curie, Section de Biologie, Campus Universitaire, 91405 Orsay Cedex, France. identified stable pre-mRNA-containing complex formed during in vitro splicing, it appears to commit the premRNA to the splicing pathway (Legrain et al. 1988; Seraphin and Rosbash 1989a).

Yeast U1 snRNA has 568 nucleotides, $\sim 400$ more than metazoan U1 snRNA (Kretzner et al. 1987; Siliciano et al. 1987). Our recent secondary structure model of yeast Ul snRNA contains two striking features: the conserved cruciform "universal core" structure, characteristic of all eukaryotic Ul snRNAs, and an extensive series of yeast-specific helices peripheral to the universal core (Kretzner et al. 1990). The yeast-specific regions include a subdomain that we have dubbed the "yeast core" because it is relatively conserved among the three yeast species that were analyzed (Kretzner et al. 1990). Its functional role, if any, is unknown.

To begin an analysis of the contribution of specific domains to U1 snRNP function, we generated yeast strains that carry mutations in two regions of Ul snRNA. The first was a highly conserved subregion of the universal core (loop II) that shows virtual identity (9 of 10) with the highly conserved "A loop" present in metazoan U1 (Kretzner et al. 1987; Siliciano et al. 1987). The second was the yeast core subdomain.

The results were surprising in that almost all of these mutations were without effect on the growth rate of these strains. We suspected that growth rate was insensitive to (perhaps even substantial) decreases in U1 snRNP activity. This interpretation was buttressed by a quantitative assessment of growth rate during U1 snRNA depletion in vivo; these data showed that U1 
snRNA levels could be reduced $>10$-fold with no detectable effect on growth rate, indicating that this assay is a very insensitive indicator of U1 snRNP activity. Indeed, the use of more sensitive assays to measure U1 snRNP activity showed that many of the Ul mutants with no growth rate phenotype decreased splicing efficiency. The results are discussed in the context of previous in vivo experiments that have addressed snRNP structurefunction relationships by genetic means.

\section{Results}

Mutations in loop II or the yeast core domain result in little or no growth phenotypes

Our secondary structure model of yeast U1 snRNA is shown in Figure 1A (Kretzner et al. 1990). Helices I-IV, as well as the ends of the molecule, constitute the universal core and resemble metazoan U1. Helices V, VII, and VIII, in addition to the proximal region of helix VI, constitute the yeast core as they are well-conserved among the three yeast species analyzed (Kretzner et al. 1990).

We targeted two regions for mutagenesis. In the universal core, we focused on loop II, the equivalent of the metazoan A loop (Fig. 1B). This highly conserved region has been shown to interact in a sequence-dependent fashion with the U1 snRNP-specific 70K protein and is of functional importance in mammalian cells (Hamm et al. 1987, 1988, 1990; Patton and Pederson 1988; Query et al. 1989; Surowy et al. 1989; Yuo and Weiner 1989). We constructed an extensive series of single-, double-, and triple-point mutants in loop II; a total of 45 mutants were recovered and sequenced. In addition, we generated a deletion of this loop 19 of 10 nucleotides; Fig. 1B legend).

In the yeast core we focused on helix VII, as it appeared to be the most conserved helix in this region and therefore a likely candidate for generating mutant phenotypes. In this region, we generated a series of triple mutants designed to test the validity of the proposed secondary structure (Kretzner et al. 1990) and its contribution to Ul snRNP function (Fig. 1C). We also generated a 95-nucleotide deletion of stem loops VII, VIII, and part of stems V, VI, IX, and X (Fig. 1A; $\Delta$ YC). This deletion, based on convenient restriction sites, removed most of the yeast core region.

The mutant Ul genes were introduced into yeast, and the wild-type U1 gene was eliminated by a standard plasmid shuffle strategy (e.g., Seraphin et al. 1988). In all cases but one, mutant strains and the isogenic wild-type control strain grew indistinguishably at all temperatures tested $\left(18^{\circ} \mathrm{C}, 25^{\circ} \mathrm{C}, 30^{\circ} \mathrm{C}\right.$, and $\left.37^{\circ} \mathrm{C}\right)$. The only exception was the deletion of the yeast core region (strain $\Delta \mathrm{yc}$ ), which grew identically to the wild-type strain at $30^{\circ} \mathrm{C}$ but grew slowly at $37^{\circ} \mathrm{C}$ (data not shown).

U1 snRNA levels can decrease at least 10-fold with no growth rate effects

The lack of a phenotype of the helix VII mutations and the mild phenotype of the $\Delta \mathrm{YC}$ deletion were consistent with analogous observations made on deletions of much of the additional sequences present in yeast U2 snRNA (Igel and Ares 1988; Shuster and Guthrie 1988). We were surprised, however, by the lack of a phenotype associated with the loop II mutations, especially the loop II deletion. We considered the possibility that this deletion, and perhaps mutations in the yeast-specific region with no growth phenotype, did affect U1 snRNP function but not sufficiently to generate a growth rate phenotype.

To estimate the level of sensitivity of the growth rate assay, we carefully measured the effect of in vivo U1 snRNA depletion (Seraphin and Rosbash 1989a) on growth rate and on U1 snRNA levels. For at least $12 \mathrm{hr}$ after U1 snRNA transcription is arrested by the addition of glucose to the GAL-U1 strain, there is no detectable effect on growth rate (Fig. 2A; Seraphin and Rosbash 1989a). During this time, the level of U1 snRNA decreases substantially (Fig. 2B). The rate of decrease is compatible with dilution of a stable U1 snRNP population, that is, by the continued wild-type growth of the culture in the virtual absence of Ul snRNA synthesis and decay. The culture maintains a normal growth rate well after the U1 snRNA level decreases below $10 \%$ of that of a wild-type strain (Fig. 2). We conclude that growth rate is an insensitive indicator of Ul snRNA levels (and U1 snRNP activity levels-assuming a single Ul snRNP population and Ul snRNP activityl and that U1 snRNA mutations must have more than a 10 -fold effect before they impact growth rate. These considerations make it very difficult to estimate or compare the strengths of nonlethal mutations on U1 snRNP activity from growth rate measurements.

\section{Effect of U1 mutations on the splicing of an inefficient pre-mRNA}

To assay pre-mRNA splicing efficiency, we initially analyzed by primer extension the levels of rp51 mRNA from strains harboring either $\triangle \mathrm{YC}$ or the wild-type $\mathrm{Ul}$ gene. rp51 mRNA levels in the mutant strain were indistinguishable from those in the wild-type strain (data not shown). On this basis, we suspected that the other U1 snRNP mutants would also have no effect by this assay. Consequently, we sought a more sensitive indicator of Ul snRNP function in vivo. For this purpose, we employed a reporter gene construct that contains an inefficiently spliced synthetic intron within a $\beta$-galactosidase-encoding open reading frame. On galactose induction, $\beta$-galactosidase is expressed from this reporter gene in a splicing-dependent fashion $\left(\mathrm{Acc}^{0}\right.$; Legrain and Rosbash 1989). This construct and its parent gene lacking an intron (pLGSD5) were transformed into a variety of loop II and helix VII mutant strains. Splicing efficiency was defined as the ratio of $\beta$-galactosidase activity generated from $\mathrm{Acc}^{0}$ to that generated from pLGSD5 in a single strain. Splicing efficiencies of various U1 mutant strains were measured and compared to that of an isogenic wild-type U1-containing strain (Fig. 3).

Of the five loop II mutants examined (Fig. 3A), the 



Figure 1. Secondary structure and mutations of the yeast UI snRNA. (A) Secondary structure of the yeast U1 snRNA. The shaded areas indicate the structures that are not well established by phylogenetic comparisons (Kretzner et al. 1990). The region removed by $\triangle \mathrm{YC}$ is shown inside the box. (B) Yeast Ul snRNA loop II and some point mutations in loop II. The numbering starts at the first nucleotide of yeast U1 snRNA. A total of 45 loop II point mutations was obtained. They included 10 single-point mutations, 23 double-point mutations, 10 triple-point mutations, 1 quadruple-point mutation, and 1 quintuple-point mutation. Overall, every nucleotide in loop II has been subjected to mutation. In addition, a 9-nucleotide deletion (from 27 to 35 ) of loop II was made ( $\Delta$ LII). All of the mutant molecules were used to transform yeast and generated viable strains with no obvious growth phenotypes. Only the point mutations that have been combined with the $\triangle Y C$ deletion are shown here; other mutant sequences are available upon request. $(C)$ Mutations in yeast Ul snRNA helix VII. HVII-ml was designed to destabilize three base-pairings proximal to loop VII. Note that HVII-m3 may destabilize only two base-pairings instead of three, because of a potential G-U pairing. The combination of HVII-ml and HVII-m 3 results in HVII-m2, which completely restores base-pairing in this region, whereas HVII-m 4 contains two triple mutants (m) and 3 changes at the opposite side of the stem) that do not complement.

loop II deletion ( $\Delta \mathrm{LII})$ had the strongest effect on $\beta$-galactosidase expression from $\mathrm{Acc}^{0}$ and reduced splicing efficiency fourfold. Two of the loop II point mutants, 30A and LII-ml (a double-point mutant $30 \mathrm{U}$ and $35 \mathrm{C}$ ), also had strong effects. Two other mutants, $27 \mathrm{~A}$ and $36 \mathrm{U}$, had higher splicing efficiencies than wild-type U1 (for unknown reasons). The data indicate that some mutants in the loop II region reduce splicing efficiency despite the absence of a growth phenotype.
We then applied this assay to the mutants in the yeast core region (Fig. $3 \mathrm{~B}$ ). In the $\triangle \mathrm{YC}$ mutant strain, splicing efficiency was reduced approximately sixfold (note that cells were grown and induced at $30^{\circ} \mathrm{C}$ where the strain showed no growth defect). Of the four mutant strains examined (see Fig. 1C), helix VII-m3 (HVII-m3) had no effect, and a slight reduction in splicing efficiency by HVII-m 1 was consistently observed. The combination of these two mutations (HVII-m2) restored base-pairing and 


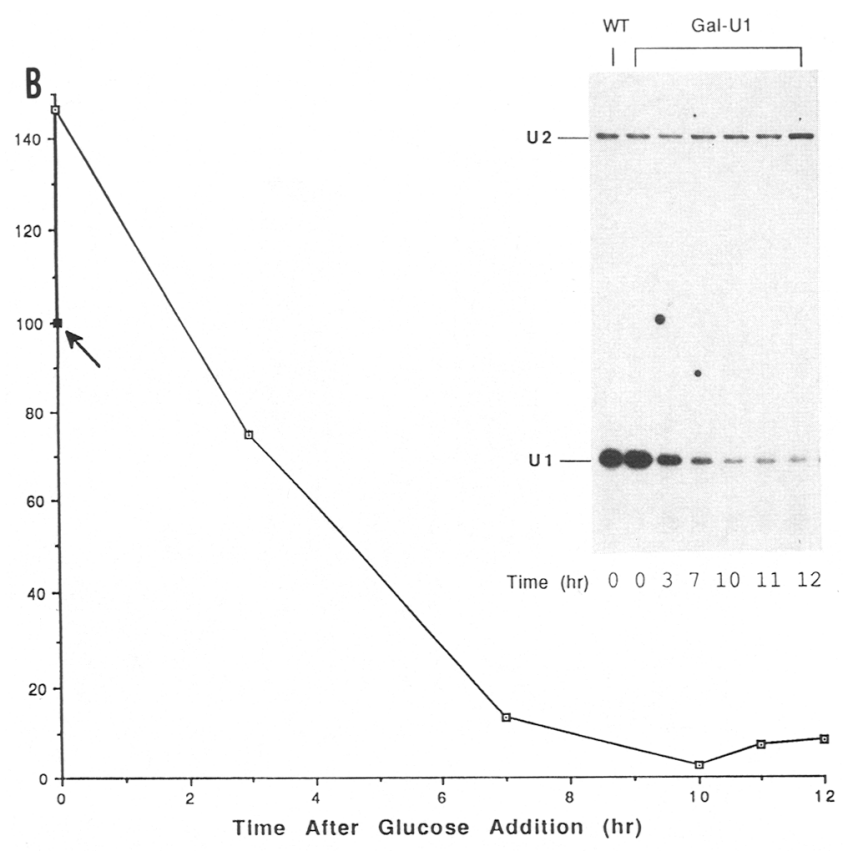

Figure 2. In vivo depletion of U1 snRNA for $12 \mathrm{hr}$ has no effect on growth rate. (A) Growth curves of the GAL-U1 strain (BS-Y82; Seraphin and Rosbash 1989a) and the isogenic wild-type strain after the shift [at time zero (0)] from galactose-containing medium to glucose-containing medium. ( $\square)$ Wild type; $\mid G A L-U 1$. (B) Time course of U1 snRNA depletion. The graph shows the relative U1 snRNA levels of the GAL-Ul strain at various time points after glucose addition. The data were determined by quantitation of the primer extension products on an AMBIS Radioanalytic Imaging System (AMBIS Systems). The U1/U2 ratio of the wild-type strain before glucose addition (time 0) was set as $100 \%$ (arrow), and those of the GAL-Ul strain at different time points were normalized accordingly. (Insert) Levels of U1 snRNA were measured before (time 0) and after addition of glucose by primer extension with DT168. Levels of U2 snRNA were measured in the same samples by primer extension with DT58 at 30 -fold lower specific activity to serve as control for loading (assuming that $\mathrm{U} 2$ snRNA level remains constant under these conditions). The strains and the time points are indicated above and below each lane, respectively.

wild-type splicing levels, whereas a nonpaired combination (HVII-m4) was more severely affected than either HVII-ml or HVII-m3. The data suggest that both the primary and secondary structure of helix VII contribute to splicing efficiency (see Discussion).

A relatively simple explanation for the low splicing efficiency of some mutant strains is that they contain a low level of Ul snRNP, that is, the mutations result in poor U1 snRNP assembly and/or a decreased half-life leading to a low steady-state level of U1 snRNP. To test this possibility, we measured by primer extension the U1 snRNA levels in the same U1 mutant strains that were assayed for $\beta$-galactosidase expression (Fig. 4). All of the loop II mutants (Fig. 4A) and the four helix VII mutant strains (Fig. 4B) were indistinguishable from the wild-type strain; however, the U1 snRNA level in the yeast core deletion strain was reduced approximately twofold. When compared to the in vivo depletion data of Figure 2, these results imply that even the yeast core deletion affects splicing efficiency by reducing U1 snRNP activity rather than U1 snRNA levels.

\section{Combinations of loop II mutants with yeast} core mutants

A likely explanation for the frequent lack of a growth phenotype is that most of the mutants do not decrease U1 snRNP activity sufficiently to reach a threshold, below which U1 snRNP activity is rate-limiting for splicing and growth. This notion suggests that the combination of a point mutant with a deletion (e.g., a loop II point mutant combined with the yeast core deletion) might manifest growth rate effects that are more severe than either mutant assayed alone. A growth rate comparison of different point mutants might then parallel their relative effects on splicing efficiency. To test this prediction, several of the loop II mutants were transferred into the $\triangle \mathrm{YC}$ background, and all of the yeast core mutants were transferred into the loop II deletion background. The ability of the various mutant combinations to support growth at different temperatures was then compared (Table 1; Fig. 5).

Addition of any of the nine loop II mutants to the $\triangle Y C$ deletion had a readily visible effect on growth (Table 1 ); none of these double mutant combinations were viable at $37^{\circ} \mathrm{C}$, thus displaying a more severe phenotype than any of the loop II mutants (normal growth at $37^{\circ} \mathrm{C}$ ) or the $\triangle \mathrm{YC}$ deletion background (slow growth at $37^{\circ} \mathrm{C}$ ). Strongest were the loop II deletion, multiple-point mutations LII-ml and LII-m3, and mutant 30A. These four mutants were unable to support growth at $30^{\circ} \mathrm{C}$ (or at $18^{\circ} \mathrm{C}$ and $25^{\circ} \mathrm{C}$ ), as well as at $37^{\circ} \mathrm{C}$. The remaining five mutants were viable at $30^{\circ} \mathrm{C}$. These data agree well with the effects of the mutants on splicing efficiency, as the loop II deletion had the strongest effect, mutants $30 \mathrm{~A}$ and LII-ml were intermediate in phenotype, and 27A and $36 \mathrm{U}$ were even "better" than wild type (Fig. 3A). 

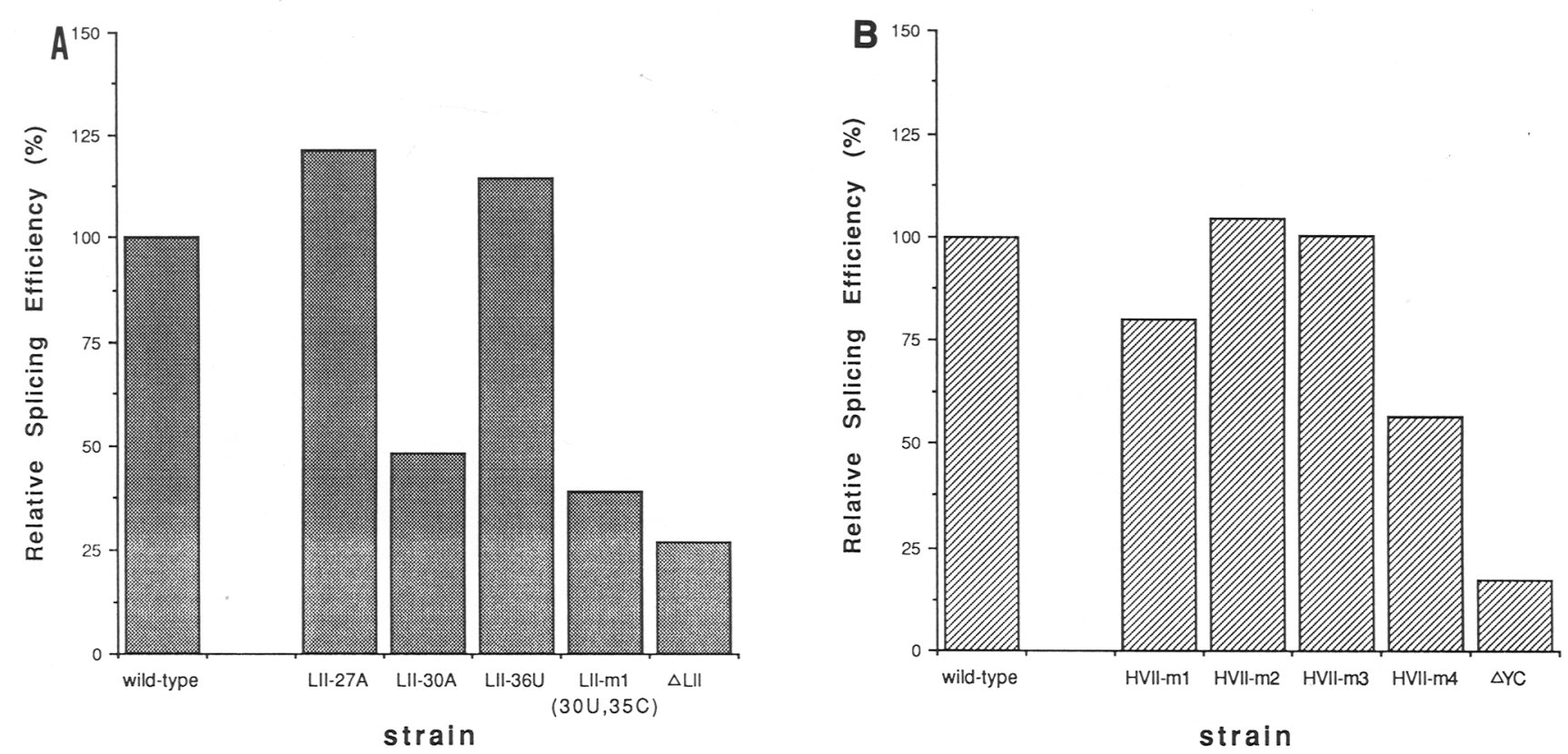

Figure 3. In vivo splicing efficiency of a reporter gene in the wild-type and mutant Ul strains. The histograms depict splicing efficiency in the mutant strains as a percentage of that in the wild-type strain. Splicing efficiency is defined as the ratio of the $\beta$-galactosidase activity of an $\mathrm{Acc}^{0}$-transformed strain to that of the same pLGSD5-transformed strain. Real values of $\beta$-galactosidase activity from pLGSD5 ranged from 1500 to 2000 units and did not show strain-specific variations. (A) Splicing efficiency in the loop II mutant strains. Data from the mutant strains were averaged from two to three experiments: LII-27A (112.7 and $130.2 \%)$; LII-30A (52.6 and $43.4 \%)$; LII-36U (119.4 and $109.3 \%)$; LII-ml (39.6 and $38.0 \%)$; and $\Delta$ LII $(24.2$ and $30.0 \%)$. (B) Splicing efficiency in the helix VII mutant strains and in the $\triangle \mathrm{YC}$ strain. Data from these mutant strains were averaged from three to four experiments: HVII-ml $/ 86.8$, $78.5,79.3$, and $77.6 \%) ;$ HVII-m2 $(95.2,103.5$, and $115.2 \%)$; HVII-m3 $(95.2,94.3,98.5$, and $114.1 \%) ;$ HVII-m4 (52.6, 46.7, 69.0, and $56.8 \%)$; and $\triangle \mathrm{YC}(18.4,18.4$, and $15.0 \%)$.

Similar results were obtained with the analysis of the helix VII mutants in a loop II deletion background (Fig. 5). Three of the four helix VII mutants (Fig. 1C), when combined with the loop II deletion, did not grow at $37^{\circ} \mathrm{C}$. The mutant HVII- $\mathrm{m} 2$ that restores Watson-Crick basepairing grew very slowly at $37^{\circ} \mathrm{C}$, noticeably better than the other three mutant strains. The phenotypes were therefore more severe than that of the loop II deletion (normal growth at $37^{\circ} \mathrm{C}$ ) or any of the four helix VII mutants assayed individually (normal growth at $37^{\circ} \mathrm{C}$. At $34^{\circ} \mathrm{C}$, the HVII-m2 strain also grew noticeably better than any of the other three combinations. At this temperature, the mutants could be easily ranked by colony size: HVII-m $2>$ HVII-m $3>$ HVII-m $1>$ HVII-m4 (Fig. 5 ). The order is consistent with that obtained from the splicing efficiency measurements (Fig. 3B) and further supports the proposed pairing in helix VII. We suggest that the presence of one deletion (either $\Delta$ LII or $\Delta Y C$ ) decreases U1 snRNP activity sufficiently so that it is rate-limiting for growth, or nearly so. In this genetic background, the presence of additional U1 snRNP mutations gives rise to growth rate decreases at elevated temperatures that reflect their effects on U1 snRNP activity. The temperature sensitivity might indicate a direct effect of temperature on mutant Ul snRNP activity (by affecting, e.g., snRNP assembly|. Alternatively, incubation at elevated temperatures might indirectly lower the Ul snRNP threshold value by increasing the requirement for Ul snRNP activity and thereby increasing the effects of the mutants on growth (see Pikielny and Rosbash 1985; Seraphin and Rosbash 1989b).

To test whether the different growth rates of the helix VII mutants in the loop II deletion background might be due to different U1 snRNP levels, we measured the steady-state U1 snRNA levels of these double-mutant combinations at $30^{\circ} \mathrm{C}$, as well as after prolonged growth at the semipermissive temperature of $34^{\circ} \mathrm{C}$ (Fig. 6). In every case, U1 RNA levels were indistinguishable from the wild-type or from the loop II deletion mutant. The results suggest that the growth phenotypes reflect differences in Ul snRNP activities rather than differences in Ul snRNA levels.

\section{Discussion}

In the experiments described in this report, we have mutagenized two regions of the yeast Ul snRNA molecule and analyzed the consequences on cell growth and premRNA splicing in vivo. One region was the helix VII subdomain of the yeast core (Kretzner et al. 1990), and the other was loop II, the yeast equivalent of the metazoan A loop.

Given the conservation of the helix VIl region, it was surprising that these mutations had little or no effect on growth rate. We were especially surprised by the absence of a growth phenotype associated with a deletion of the highly conserved loop II. Taken alone, this result would suggest that yeast does not have a U1 70K-like protein, 


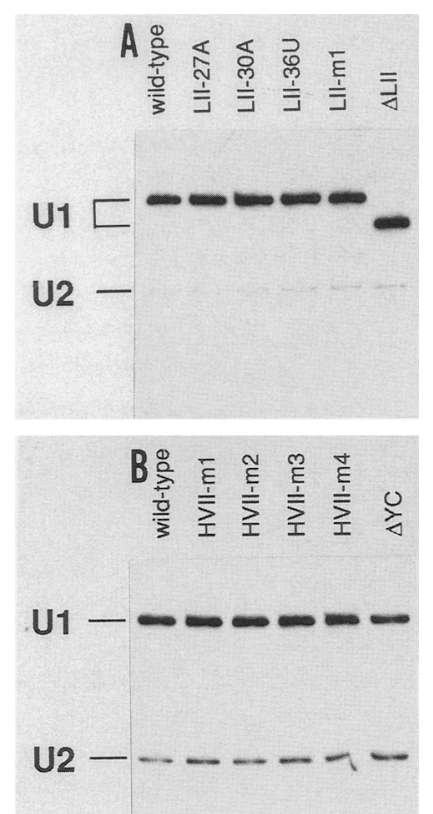

Figure 4. Steady-state levels of Ul snRNA from wild-type and mutant strains. (A) Steady-state levels of U1 snRNA from wildtype and loop II mutant strains (shown above each lane) were assayed by primer extension with DT586. The U2 primer DT58 was used at fivefold lower specific activity. (B) Steady-state levels of Ul snRNA from wild-type, helix VII mutants, and $\triangle \mathrm{YC}$ strains (shown above each lane) were assayed by primer extension with DT586.

that the protein is not important for yeast Ul snRNP function, or that other molecular interactions (proteinprotein or protein-RNA) are sufficient to retain this protein in the snRNP. Two groups have also shown that mammalian U2 snRNP can function despite a deletion of a known protein-binding region (Hamm et al. 1989; Pan and Prives 1989). In any case, the absence of a growth phenotype for the loop II deletion caused us to consider other interpretations of the negative or mild phenotypes also associated with the yeast core mutations.

We suspected that some of the mutations affected U1 snRNP function, perhaps substantially, but that the effects were not sufficient to generate a growth phenotype. The Ul depletion data suggest that one might be able to define a threshold value (at least 10), indicating the extent of Ul snRNP level or activity decrease required to generate a growth rate phenotype. Less quantitative data for U2 snRNP depletion (Seraphin and Rosbash 1989a) and for U5 snRNP depletion (Patterson and Guthrie 1987; data not shown) suggest that a high threshold value is probably not restricted to Ul snRNP. Perhaps a high snRNP level is required for optimal splicing efficiency under other physiological conditions or for the splicing of pre-mRNAs that do not affect growth rate.

Consistent with the idea that these mutations affect U1 snRNP function despite the absence of a growth phenotype, several mutants had effects on $\beta$-galactosidase expression from an inefficiently spliced reporter gene construct (Fig. 3). Also, mutant combinations (combina-
Table 1. Growth phenotypes of the loop II mutants in the $\triangle Y C$ background

\begin{tabular}{|c|c|c|}
\hline \multirow[b]{2}{*}{ Mutants $^{\mathrm{a}}$} & \multicolumn{2}{|c|}{ Growth $\left({ }^{\circ} \mathrm{C}\right)^{\mathrm{b}}$} \\
\hline & 30 & 37 \\
\hline LII (control) & + & $+($ slow $)$ \\
\hline LII-27A & + & - \\
\hline LII-27U & + & - \\
\hline LII-28U & + & - \\
\hline LII-30A & - & - \\
\hline LII-36U & + & - \\
\hline LII-m l $\left(\begin{array}{l}300 \\
35 \mathrm{C}\end{array}\right)$ & - & - \\
\hline $\begin{array}{r}\text { LII-m2 }\left(\begin{array}{l}33 \mathrm{C} \\
34 \mathrm{U}\end{array}\right) \\
(27 \mathrm{~A})\end{array}$ & + & - \\
\hline LII-m3 $\left(\begin{array}{l}29 G \\
35 G\end{array}\right)$ & - & - \\
\hline$\Delta \mathrm{LII}$ & - & - \\
\hline
\end{tabular}

${ }^{a}$ Changes (or no change in the case of the control) in yeast loop II that were placed in the $\triangle \mathrm{YC}$ background. Strains that were unable to grow at $30^{\circ} \mathrm{C}$ were also unable to grow at $25^{\circ} \mathrm{C}$ and $18^{\circ} \mathrm{C}$.

bPlus $(+)$ indicates growth; minus $|-|$ indicates no growth at the temperatures shown.

tions of point mutants or multiple-point mutants in one region with a deletion in the other) had much stronger growth phenotypes than either mutant alone. The latter results are analogous to observations made in selfsplicing RNAs, where mutations of phylogenetically important nucleotides had no phenotype; however, when placed in an already mutant background, they enhanced dramatically the initial mutant phenotype (Michel and Jacquier 1987).

These assays allowed us to compare in two independent ways the effects of different point mutants and

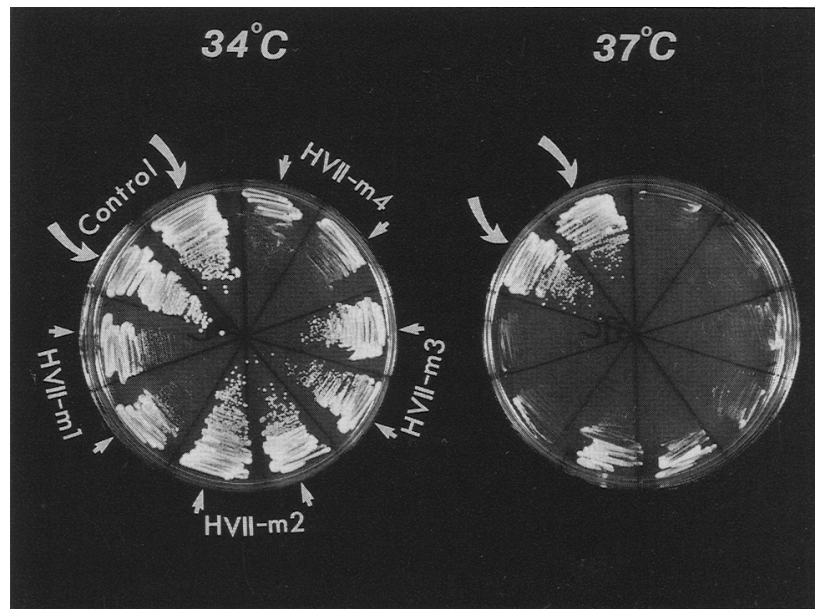

Figure 5. Growth phenotypes of helix VII mutants in the loop II deletion background. Two independent transformants of each strain were analyzed on YM-1 plates incubated at $34^{\circ} \mathrm{C}$ and $37^{\circ} \mathrm{C}$. Two independent transformants of the parent loop II deletion strain (control) were analyzed in parallel (large arrows). The names by one plate $\left(34^{\circ} \mathrm{C}\right)$ indicate the helix VII mutations that were combined with $\Delta \mathrm{LII}$; the orientation of strains is the same on the other plate. 




Figure 6. Steady-state levels of Ul snRNA from double-mutant combinations that contain helix VII mutations in the loop II deletion background. Cells were grown overnight at $30^{\circ} \mathrm{C}$ (permissive temperature) and at $34^{\circ} \mathrm{C}$ /where the growth phenotypes for the various strains are best distinguished) before RNA extraction. DT586 was used to prime Ul snRNA, and DT58 at fivefold reduced specific activity was used to prime U2 snRNA. The names of individual strains are shown above each lane.

multiple-point mutants within a single region. In the loop II region, the 9-nucleotide deletion had the most severe phenotype for splicing and for growth rate when combined with the yeast core deletion. The data summarized in Table 1 indicate that the mutant $30 \mathrm{~A}$ was the most severe of six transversions assayed by growth rate (mutants at positions 27, 28, 30,33,34,36; positions 33 and 34 were assayed together in mutant LII-m2 which grew better than mutant 30A). Although less extensive, the $\beta$-galactosidase data (Fig. $3 \mathrm{~A}$ ) are consistent with this conclusion. It is interesting to note that nucleotide 30 corresponds to the location of the most severe point mutant among those assayed in the human U1 snRNA A loop (position 31 in Yuo and Weiner 1989).

In the yeast core region, the large deletion had the most severe effect on $\beta$-galactosidase activity and the most severe growth rate effect when combined with the loop II deletion. Of the four helix VII mutants (Fig. 1C), the compensatory triple mutant combination (HVII-m2) had the least severe effect on $\beta$-galactosidase activity (Fig. 3B) and the least severe effect on growth when assayed in a loop II deletion background (Fig. 5). The noncompensatory triple mutant combination (HVII-m4) had the most severe effect on $\beta$-galactosidase activity (Fig. 3B) and the most severe effect on growth in a loop II deletion background (Fig. 5). Both of these assays are consistent with some of the proposed base-pairing in helix VII (Kretzner et al. 1990), as the effects of mutant HVII-ml (especially apparent in the growth rate assay) are clearly relieved by the additional complementary mutations. However, in vivo studies of this kind cannot distinguish between a positive effect of the correct secondary structure and a negative effect of the incorrect structure on Ul snRNP function.

The data also suggest that in addition to secondary structure, the primary sequence of the mutated nucleo- tides affect U1 snRNP activity. This is because the compensatory mutant combination (HVII-m2) grows less well than the control, despite a similar number of base pairs in helix VII. Also, the noncompensatory combination (HVII-m4) grows less well than either HVII-ml or HVII-m3; in all three cases, the bottom 3 bp of helix VII is disrupted /although HVII-m3 can form a single G-U pair in this region). A contribution by both the primary and secondary structure of helix VII is consistent with our previous structural analysis (Kretzner et al. 1990).

Our data also indicate that the effects of the loop II mutants and the helix VII mutants on Ul snRNP activity are not an indirect consequence of changes in $\mathrm{U} 1$ snRNA levels. The absence of an effect on U1 snRNA levels is in contrast to the observations of Yuo and Weiner (1989), who showed that some mutations in the human Ul snRNA A loop decreased mutant Ul snRNA levels. Destabilization of U1 snRNA by loop II mutations in the HeLa system may reflect differences between yeast and metazoan snRNP assembly. Alternatively, destabilization could be caused by competition between endogenous wild-type and transiently expressed mutant snRNAs for a limited supply of snRNP proteins. Recent experiments in the Xenopus system (Hamm et al. 1990) make this latter interpretation more likely.

Because we have not undertaken any cell fractionation, it cannot be excluded that a large fraction of the mutant snRNAs are cytoplasmic and that the nuclear U1 snRNP levels are significantly decreased. We suggest, however, that these mutants have a normal level of nuclear Ul snRNP with a reduced or altered U1 snRNP activity, perhaps as a consequence of a qualitative defect in Ul snRNP assembly. The mutations may cause the absence or mispositioning of U1 snRNP proteins (e.g., a yeast equivalent of the mammalian $70 \mathrm{~K}$ protein in the case of the loop II mutants). Alternatively, a proper loop II sequence and helix VII sequence and structure may participate directly in U1 snRNP function.

The use of inefficiently spliced substrates and the analysis of double mutant combinations indicate that both the universally conserved loop II sequence and at least part of the yeast core are important for optimal splicing efficiency. Presumably, the lack of a growth phenotype for many of these mutants indicates that there is essentially no effect on mRNA levels for many intron-containing genes. This is based on substantial evidence that demonstrates growth rate decreases due to even modest reductions in mRNA levels from introncontaining ribosomal protein genes (e.g., Abovich and Rosbash 1984).

The lack of an effect on mRNA levels could indicate that reduction in splicing efficiency is still not rate-limiting for gene expression (Pikielny and Rosbash 1985; Seraphin and Rosbash 1989b). Alternatively, the mutants may cause no reduction in splicing efficiency because U1 snRNP function (or the aspect of U1 snRNP function affected by the mutations) is not rate-limiting for splicing under these growth conditions. A consequence of the latter possibility is that mutations in re- 
gions involved in splicing may have little or no biochemical phenotype, as well as no growth phenotype, when assayed under normal or near normal conditions. This may explain the mild effects of the helix VII mutants on $\beta$-galactosidase activity (Fig. 3B). Our results underscore the importance of using inefficient substrates and making double-mutant combinations in these kinds of in vivo studies. It will be interesting to see whether similar structural and functional studies on the "extra" sequences in yeast U2 snRNA give rise to similar results.

We imagine two possibilities to explain the enhancement of growth rate effects observed in the mutant combinations analyzed in this study. The first is that the loop II region and associated proteins physically interact (perhaps indirectly) with the yeast core region. A mutant loop II region would therefore enhance the structural consequences of the $\triangle \mathrm{YC}$ deletion, and vice versa. Because the levels of the double mutant RNAs are normal, the putative interaction must impact on Ul snRNP activity (perhaps through a qualitative effect on proper U1 snRNP assembly) rather than on the rate of U1 snRNP synthesis or turnover. The second, more general possibility is that one deletion lowers Ul snRNP activity and makes its contribution to splicing rate-limiting for growth, or nearly so. This makes the cell sensitive to the presence of additional Ul snRNP mutations that further reduce, even modestly, Ul snRNP activity. We favor this second possibility because it does not require any ad hoc assumptions about physical interactions between parts of the molecule.

The conservation of the yeast core domain among different yeast species and its absence from metazoan U1 snRNA (Kretzner et al. 1990) reinforced the possibility that there may be some mechanistic differences between yeast and mammalian pre-mRNA splicing. The effects of the helix VII mutations on U1 snRNP activity make this possibility more likely. In vitro experiments will be required to verify this prediction and to determine whether these differences are quantitative or qualitative in nature.

\section{Materials and methods}

Strains and plasmids

Plasmid pTZSNR19-SaII contains, in a pTZ19U backbone, an EcoRI-HindIII fragment encompassing the SNR19 gene ( $S$ cerevisiae Ul gene) (Kretzner et al. 1987; Seraphin et al. 1988). A Sall site was introduced by linker insertion at the HindIII site. A deletion was made that removed 5'-flanking DNA from the EcoRI site to an EcoRV site at -360 to generate plasmid pXL7. A PvuII-Sall fragment containing the above U1 clone from pXL7 was subcloned to a plasmid that has a CEN3 (BamHI fragment) in YRp7 (Struhl et al. 1979; Seraphin et al. 1988) to give rise to $\mathrm{pXL} 8$. Therefore, $\mathrm{pXL8}$ is the same as the previously described pBS46 (Seraphin and Rosbash 1989a), except for the shortened 5 '-flanking sequences. It has an identical phenotype to pBS46 when introduced into yeast (data not shown).

For construction of the yeast core deletion $(\triangle \mathrm{YC}), \mathrm{pBS} 46$ was cut with ApaI and BsmI, treated with T4 DNA polymerase to create blunt ends, and self-ligated. Sequencing of the deletion revealed that two additional nucleotides had been removed at the ApaI site. The exact sequence of $\triangle Y C$ is shown in Figure 1A. Deletion and point mutations of loop II were made by oligonucleotide-directed mutagenesis of the U1 gene in either pTZSNR19-SalI or pXL7, using three different methods interchangeably (Kunkel 1985; Nakamaye and Eckstein 1986; Hill et al. 1987). All point mutants and deletions were confirmed by sequencing (Sanger et al. 1977) and were subcloned such that the resulting yeast-Escherichia coli shuttle plasmids are identical to pXL8 except for the specified mutations. Mutations in the helix VII of yeast U1 snRNA were made by the PCR (polymerase chain reaction/ technique, using mutant oligonucleotides and pXL8 as the template. DNA fragments from the PCR were subcloned to replace the wild-type fragment in pXL8. Sequencing across the subcloned junctions and throughout the PCR fragments revealed no other changes except the intended ones. Combinations of the $\triangle Y C$ deletion with the loop II mutations (and the loop II deletion with the helix VII mutations) were made by switching fragments from two plasmids between a StyI site in the U1 gene and a PvuII site in the yeast vector.

Yeast transformation of BS-Y20 (MATaleu2-3 leu2-112 ura3-52 trp1-289 arg4 ade2 snr19 :: LEU2 p23) and selection of mitotic segregants that have lost p23 were as described (Seraphin and Rosbash 1989a). Growth curve analysis and U1 snRNA depletion were carried out as described by using strains BS-Y82 (GAL-U1 strain) and BS-Y46 [isogenic to BS-Y82 but with the promoter of $\mathrm{U} 1$ instead of the $G A L$ upstream activating sequence (UAS); Seraphin and Rosbash 1989a]. Plasmids used in the $\beta$-galactosidase assays (pLGSD5, $A c^{\circ}$ ) were described by Legrain and Rosbash (1989).

\section{$\beta$-Galactosidase assays}

Induction of the reporter genes (for $3 \mathrm{hr}$ ) and $\beta$-galactosidase assays were as described (Legrain and Rosbash 1989).

\section{RNA extraction and primer extension}

RNA extraction and primer extension were carried out according to published procedures (Pikielny and Rosbash 1985). Primer DT58 is complementary to positions $100-121$ of yeast U2 snRNA. Primers DT168 and DT586 are complementary to positions $27-44$ and $131-149$ of yeast U1 snRNA, respectively. Extension products were analyzed on $5 \%$ or $6 \%$ polyacrylamide denaturing gels.

\section{Acknowledgments}

We thank Jeff D. Harrison for assistance with $\beta$-galactosidase assays, C.W. Pikielny, H.V. Colot, A.M. Weiner, and I. Edery for their suggestions and comments on the manuscript, and L.J. Lorenz for help with the figures. We extend special thanks to $T$. Tishman for expert secretarial assistance. This work was supported by grant GM-23549 to M.R. from the National Institutes of Health.

The publication costs of this article were defrayed in part by payment of page charges. This article must therefore be hereby marked "advertisement" in accordance with 18 USC section 1734 solely to indicate this fact.

\section{References}

Abovich, N. and M. Rosbash. 1984. Two genes for ribosomal protein 51 of Saccharomyces cerevisiae complement and contribute to the ribosomes. Mol. Cell. Biol. 4: 1871-1879.

Brody, E. and J. Abelson. 1985. The "spliceosome": Yeast premessenger RNA associates with a $40 \mathrm{~S}$ complex in a 
splicing-dependent reaction. Science 288: 963-967.

Cheng, S.C. and J. Abelson. 1987. Spliceosome assembly in yeast. Genes Dev. 1: 1014-1027.

Frendewey, D. and W. Keller. 1985. The stepwise assembly of a pre-mRNA splicing complex requires U-snRNPs and specific intron sequences. Cell 42: 355-367.

Grabowski, P.J., S.R. Seiler, and P.A. Sharp. 1985. A multicomponent complex is involved in the splicing of messenger RNA precursors. Cell 42: 345-353.

Guthrie, C. and B. Patterson. 1988. Spliceosomal snRNAs. Annu. Rev. Genet. 22: 387-419.

Hamm, J., N.A. Dathan, and I.W. Mattaj. 1989. Functional analysis of mutant Xenopus U2 snRNAs. Cell 59: 159-169.

Hamm, J., M. Kazmaier, and I.W. Mattaj. 1987. In vitro assembly of U1 snRNPs. EMBO $/$. 6: 3479-3485.

Hamm, J., N.A. Dathan, D. Scherly, and I.W. Mattaj. 1990. Multiple domains of Ul snRNA, including Ul specific protein binding sites, are required for splicing. EMBO I. 9: 12371244.

Hamm, J., V.L. van Santen, R.A. Spritz, and I.W. Mattaj. 1988. Loop I of U1 small nuclear RNA is the only essential RNA sequence for binding of specific Ul small nuclear ribonucleoprotein particle proteins. Mol. Cell. Biol. 8: 4787-4791.

Hill, D.E., A.R. Oliphant, and K. Struhl. 1987. Mutagenesis with degenerate oligonucleotides: An efficient method for saturating a defined DNA region with base pair substitutions. Methods Enzymol. 155: 558-568.

Igel, A.H. and M. Ares, Jr. 1988. Internal sequences that distinguish yeast from metazoan U2 snRNA are unnecessary for pre-mRNA splicing. Nature 334: 450-453.

Konarska, M.M. and P.A. Sharp. 1986. Electrophoretic separation of complexes involved in the splicing of precursors to mRNAs. Cell 46: 845-855.

- 1987. Interaction between snRNP particles in formation of spliceosomes. Cell 49: 763-774.

Kretzner, L., A. Krol, and M. Rosbash. 1990. Saccharomyces cerevisiae U1 small nuclear RNA secondary structure contains both universal and yeast-specific domains. Proc. Natl. Acad. Sci. 87: 851-855.

Kretzner, L., B.C. Rymond, and M. Rosbash. 1987. S. cerevisiae U1 RNA is large and has limited primary sequence homology to metazoan U1 snRNA. Cell 50: 593-602.

Kunkel, T.A. 1985. Rapid and efficient site-specific mutagenesis without phenotypic selection. Proc. Natl. Acad. Sci. 82: 488-492.

Lamond, A.I., M.M. Konarska, P.J. Grabowski, and P.A. Sharp. 1988. Spliceosome assembly involves the binding and release of U4 small nuclear ribonucleoprotein. Proc. Natl. Acad. Sci. 85: 411-415.

Legrain, P. and M. Rosbash. 1989. Some cis- and trans-acting mutants for splicing target pre-mRNA to the cytoplasm. Cell 57: 573-583.

Legrain, P., B. Seraphin, and M. Rosbash. 1988. Early commitment of yeast pre-mRNA to the spliceosome pathway. Mol. Cell. Biol. 8: $3755-3760$

Maniatis, T. and R. Reed. 1987. The role of small nuclear ribonucleoprotein particles in pre-mRNA splicing. Nature 325: 673-678.

Michel, F. and A. Jacquier. 1987. Long-range intron-exon and intron-intron pairings involved in self-splicing of class II catalytic introns. Cold Spring Harbor Symp. Quant. Biol. 52: $201-212$.

Nakamaye, K.L. and F. Eckstein. 1986. Inhibition of restriction endonuclease Ncil cleavage by phosphorothioate groups and its application to oligonucleotide-directed mutagenesis. $\mathrm{Nu}$ cleic Acids Res. 14: 9679-9698.
Pan, Z.-Q. and C. Prives. 1989. U2 snRNA sequences that bind U2-specific proteins are dispensable for the function of U2 snRNP in splicing. Genes Dev. 3: 1887-1898.

Patterson, B. and C. Guthrie. 1987. An essential yeast snRNA with a U5-like domain is required for splicing in vivo. Cell 49: 613-624.

Patton, J.R. and T. Pederson. 1988. The $M_{\mathrm{r}} 70,000$ protein of the U1 small nuclear ribonucleoprotein particle binds to the $5^{\prime}$ stem-loop of U1 RNA and interacts with Sm domain proteins. Proc. Natl. Acad. Sci. 85: 747-751.

Pikielny, C.W. and M. Rosbash. 1985. mRNA splicing efficiency in yeast and the contribution of nonconserved sequences. Cell 41: 119-126.

Pikielny, C.W., B.C. Rymond, and M. Rosbash. 1986. Electrophoresis of ribonucleoproteins reveals an ordered pathway of yeast splicing complexes. Nature 324: 341-345.

Query, C.C., R.C. Bentley, and J.D. Keene. 1989. A specific 31nucleotide domain of U1 RNA directly interacts with the $70 \mathrm{~K}$ small nuclear ribonucleoprotein component. Mol. Cell. Biol. 9: 4872-4881.

Ruby, S.W. and J. Abelson. 1988. An early hierarchic role of U1 small nuclear ribonucleoprotein in spliceosome assembly. Science 242: 1028-1035.

Sanger, F., S. Nicklen, and A.R. Coulson. 1977. DNA sequencing with chain-terminating inhibitors. Proc. Natl. Acad. Sci. 74: 5463-5467.

Seraphin, B. and M. Rosbash. 1989a. Identification of functional U1 snRNA-pre-mRNA complexes committed to spliceosome assembly and splicing. Cell 59: 349-358.

. 1989b. Mutational analysis of the interactions between Ul small nuclear RNA and pre-mRNA of yeast. Gene 82: 145-151.

Seraphin, B., L. Kretzner, and M. Rosbash. 1988. A U1 snRNA : Pre-mRNA base pairing interaction is required early in yeast spliceosome assembly but does not uniquely define the 5' cleavage site. EMBO I. 7: 2533-2538.

Shuster, E.O. and C. Guthrie. 1988. Two conserved domains of yeast U2 snRNA are separated by 945 nonessential nucleotides. Cell 55: $41-48$.

Siliciano, P.G. and C. Guthrie. 1988. 5' Splice site selection in yeast: Genetic alterations in base-pairing with U1 reveal additional requirements. Genes Dev. 2: 1258-1267.

Siliciano, P.G., M.H. Jones, and C. Guthrie. 1987. Saccharomyces cerevisiae has a U1-like small nuclear RNA with unexpected properties. Science 237: 1484-1487.

Steitz, J.A., D.L. Black, V. Gerke, K.A. Parker, A. Kramer, D. Frendewey, and W. Keller. 1988. Functions of the abundant U-snRNPs. In Structure and function of major and minor small nuclear ribonucleoprotein particles (ed. M.L. Birnstiel), pp. 115-154. Springer-Verlag, Berlin/Heidelberg/New York/London/Paris/Tokyo.

Struhl, K., D.T. Stinchcomb, S. Scherer, and R.W. Davis. 1979. High-frequency transformation of yeast: Autonomous replication of hybrid DNA molecules. Proc. Natl. Acad. Sci. 76: 1035-1039.

Surowy, C.S., V.L. van Santen, S.M. Scheib-Wixted, and R.A. Spritz. 1989. Direct, sequence-specific binding of the human U1-70K ribonucleoprotein antigen protein to loop I of U1 small nuclear RNA. Mol. Cell. Biol. 9: 4179-4186.

Yuo, C.-Y. and A.M. Weiner. 1989. Genetic analysis of the role of human U1 snRNP in mRNA splicing. I. Effect of mutations in the highly conserved stem-loop I of U1. Genes Dev. 3: 697-707.

Zhuang, Y. and A.M. Weiner. 1986. A compensatory base change in U1 snRNA suppresses a $5^{\prime}$ splice site mutation. Cell 46: 827-835. 


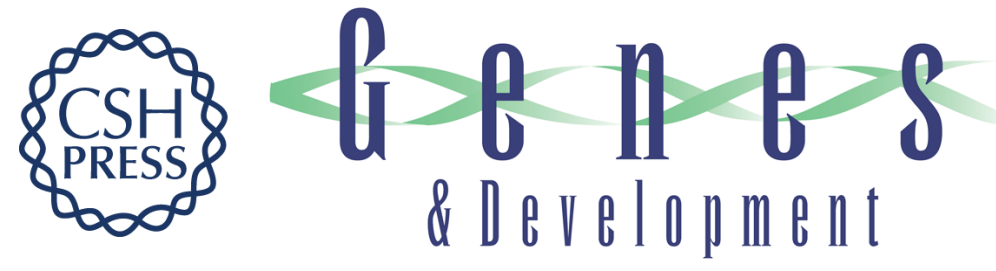

\section{Universally conserved and yeast-specific U1 snRNA sequences are important but not essential for U1 snRNP function.}

$X$ L Liao, L Kretzner, B Seraphin, et al.

Genes Dev. 1990, 4:

Access the most recent version at doi:10.1101/gad.4.10.1766

References This article cites 41 articles, 18 of which can be accessed free at:

http://genesdev.cshlp.org/content/4/10/1766.full.html\#ref-list-1

License

Email Alerting

Service

Receive free email alerts when new articles cite this article - sign up in the box at the top right corner of the article or click here.

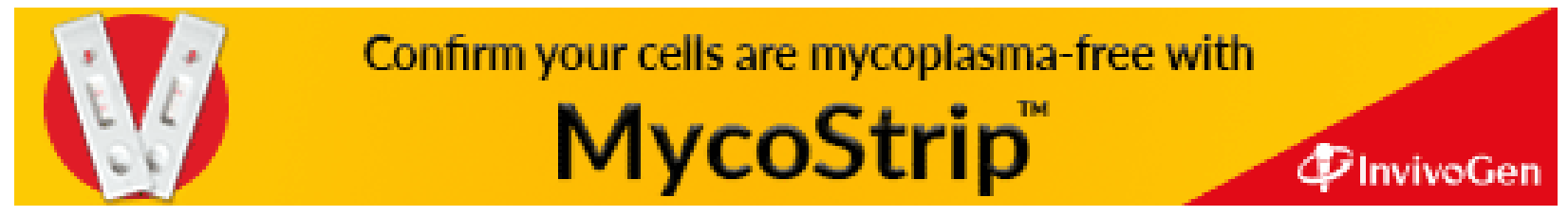

